\title{
Vibration Analysis of Inclined Laminated Composite Beams under Moving Distributed Masses
}

\author{
E. Bahmyari, S. R. Mohebpour, and P. Malekzadeh \\ Department of Mechanical Engineering, Persian Gulf University, Bushehr 75168, Iran \\ Correspondence should be addressed to E. Bahmyari; ebahmyari@yahoo.com
}

Received 17 November 2012; Accepted 14 June 2013; Published 10 February 2014

Academic Editor: Nuno Maia

Copyright (C) 2014 E. Bahmyari et al. This is an open access article distributed under the Creative Commons Attribution License, which permits unrestricted use, distribution, and reproduction in any medium, provided the original work is properly cited.

\begin{abstract}
The dynamic response of laminated composite beams subjected to distributed moving masses is investigated using the finite element method (FEM) based on the both first-order shear deformation theory (FSDT) and the classical beam theory (CLT). Six and ten degrees of freedom beam elements are used to discretize the CLT and FSDT equations of motion, respectively. The resulting spatially discretized beam governing equations including the effect of inertial, Coriolis, and centrifugal forces due to moving distributed mass are evaluated in time domain by applying Newmark's scheme. The presented approach is first validated by studying its convergence behavior and comparing the results with those of existing solutions in the literature. Then, the effect of incline angle, mass, and velocity of moving body, layer orientation, load length, and inertial, Coriolis, and centrifugal forces due to the moving distributed mass and friction force between the beam and the moving distributed mass on the dynamic behavior of inclined laminated composite beams are investigated.
\end{abstract}

\section{Introduction}

The composite beams have been increasingly used over the past few decades in the field of aerospace and civil and mechanical engineering due to their excellent engineering features. They are generally used as structural components of light-weight heavy load-bearing elements because of the high strength and stiffness-to-weight ratios, the ability of being different strengths in different directions, and the nature of being tailored to satisfy design requirements of strength and stiffness in practical designs. Hence, the better understanding of vibration characteristics of these structural elements under dynamic loads is essential for their engineering design and manufacture.

The dynamic behavior of isotropic beams subjected to moving loads using the analytical and numerical methods has been investigated by some researchers; see, for examples, [1-4]. In most of these works, the horizontal beams under moving loads have been considered and the effects of the Coriolis and the centrifugal forces have been neglected. In an interesting work, $\mathrm{Wu}[5]$ studied the vibration of an inclined isotropic beam under moving mass by considering the effects of the Coriolis and the centrifugal forces. On the other hand, little attentions have been paid to dynamic response of the laminated composite beams subjected to the moving loads.

Chandrashekhara et al. presented an exact solution for the free vibration of symmetrically laminated composite beams using first-order shear deformation and rotary inertia has been included in their analysis [6]. The solution procedure is applicable to arbitrary boundary conditions. Kadivar and Mohebpour studied the dynamic response of an unsymmetric laminated composite beam subjected to moving loads $[7,8]$. They developed a finite element method based on the third-order shear deformation theory. Law and Zhu studied the dynamics of damaged reinforced concrete bridge structures under moving vehicular loads [9]. Lee and Yhim studied the dynamics of single- and two-span continuous composite plate structures subjected to multimoving loads [10]. Kiral et al. studied the dynamic behavior of the laminated composite beams subjected to a single force traveling at a constant velocity using a three-dimensional finite element model based on the classical lamination theory [11]. Yang et al. presented an analytical study on the free and forced vibration of inhomogeneous Euler-Bernoulli beams containing open 


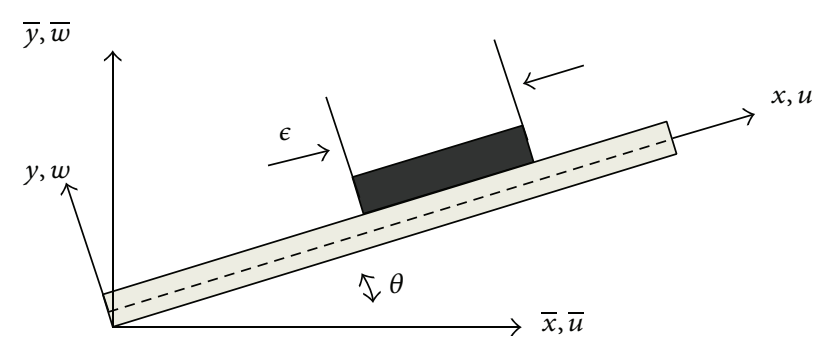

(a)

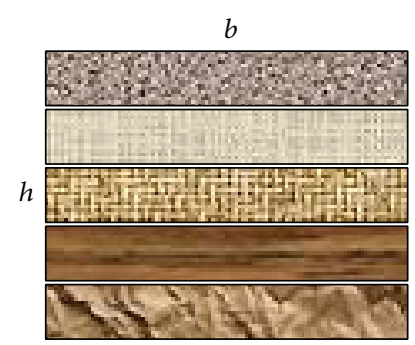

(b)

Figure 1: (a) Geometry of the problem and (b) beam cross section.

edge cracks under an axial compressive force and a concentrated transverse load moving along the longitudinal direction [12]. Şimşek and Kocatürk investigated free vibration characteristics and the dynamic behavior of a functionally graded simply supported Euler-Bernoulli beam under a concentrated moving harmonic load [13]. Malekzadeh et al. studied the dynamic response of thick laminated annular sector plates with simply supported radial edges subjected to a radially distributed line load moving along the circumferential direction by using a three-dimensional hybrid method composed of series solution, the layerwise theory, and the differential quadrature method in conjunction with the finite difference method [14]. Şimşek studied vibration of a functionally graded (FG) simply supported beam due to a moving mass by using Euler-Bernoulli, Timoshenko, and the third-order shear deformation beam theories [15]. Khalili et al. presented a mixed method to study the dynamic behavior of functionally graded (FG) beams subjected to moving loads. The theoretical formulations are based on Euler-Bernoulli beam theory, and the governing equations of motion of the system are derived using the Lagrange equations [16]. Mohebpour et al. studied the dynamic behavior of laminated composite plates traversed by a moving oscillator using the finite element method [17]. Mohebpour et al. studied the dynamic response of the laminated composite beams subjected to the moving oscillator using the first-order shear deformation theory [18]. Kahya studied the dynamic response of laminated composite beams under moving loads using the finite element method [19]. Yas and Heshmati investigated the dynamic response of functionally graded nanocomposite beams reinforced by randomly oriented carbon nanotube under the action of moving load [20]. Şimşek et al. studied dynamic behavior of an axially functionally graded beam under the action of a moving harmonic load [21]. Mamandi and Kargarnovin investigated nonlinear vibrations analysis of an inclined pinned-pinned self-weight Timoshenko beam made of linear, homogenous, and isotropic material with constant cross section and finite length subjected to a traveling mass/force with constant velocity [22].

To the best of authors' knowledge, the dynamic behavior of the inclined laminated composite beams subjected to moving distributed mass has not been investigated so far. This motivates the authors to study the problem considered here. The aim of this study is to examine the finite element dynamic analysis of the straight and the inclined composite laminated beams subjected to moving distributed mass with constant speed based on the classical (CLBT) and the first-order (FSDT) laminated beam theories. The algorithm developed here also accounts the shear deformation and the rotary inertia for the beam and centrifugal and Coriolis forces due to moving distributed mass. In addition, the friction force between the inclined beam and the moving distributed mass is considered as a uniform distributed load. The time variable is evaluated by using Newmark's scheme [18].

\section{Governing Equations}

Consider an inclined laminated composite beam of the length $L$, width $b$, thickness $h$, and incline angle $\theta$ as shown in Figure 1. The coordinate system is placed at the midplane of the laminate. The thickness of all orthotropic layers is assumed to be equal. The fibers direction is indicated by the angle $\alpha$ measuring from positive $x$-axes in counterclockwise direction. The displacement components of an arbitrary material point along the $x$ - and $z$-directions are denoted as $u(x, z, t)$ and $w(x, z, t)$, respectively.

In this paper, the following assumptions are adopted.

(i) Inertial effects of both the beam and the moving mass are taken into account as well as the gravity effects of the load.

(ii) Friction force between the moving mass and the beam is taken into account as a uniform distributed load.

(iii) The load moves at constant velocity and keeps in contact with the beam at all times.

(iv) The beam is assumed to carry the whole load at the left-hand support when the motion just started.

(v) The Coriolis and the centrifugal forces induced by the moving distributed mass are taken into account.

(vi) Poisson effect is neglected except when it is mentioned.

The governing equations can be derived using Hamilton's principle,

$$
\int_{t_{1}}^{t_{2}}(\delta U-\delta W-\delta T) d t,
$$

where $\delta U$ and the $\delta T$ denote variation of strain energy of beam and $\delta W$ is work done by external loads. Based on the 
classical beam theory, the variation of the strain energy and the kinetic energy becomes

$$
\begin{gathered}
\delta U=b \int_{0}^{l} \int_{-h / 2}^{h / 2} \sigma_{x x}\left(\delta \varepsilon_{x x}^{(0)}-z \delta \varepsilon_{x x}^{(1)}\right) d z d x \\
\delta T=b \int_{0}^{l} \int_{-h / 2}^{h / 2} \rho_{0}\left[\left(\frac{\partial u}{\partial t}-z \frac{\partial^{2} w}{\partial t \partial x}\right)\left(\frac{\partial \delta u}{\partial t}-z \frac{\partial^{2} \delta w}{\partial t \partial x}\right)\right. \\
\left.+\frac{\partial w}{\partial t} \frac{\partial \delta w}{\partial t}\right] d z d x
\end{gathered}
$$

where $\sigma_{x x}$ is the axial stress in the laminated beam and $\rho_{0}$ is the mass density of the beam. The strain components are related to the displacement components as

$$
\delta \varepsilon_{x x}^{(0)}=\delta\left(\frac{\partial u}{\partial x}\right), \quad \delta \varepsilon_{x x}^{(1)}=\delta\left(\frac{\partial^{2} w}{\partial x^{2}}\right),
$$

where $w$ and $u$ are the transverse and the axial displacements of a material point on the midplane in the $z$-and $x$-directions, respectively.

The virtual work of the external loads is as follows:

$$
\delta W=\int_{0}^{l}(q \delta w+f \delta u) d x
$$

where $q$ is the transverse distributed force on the surface of the laminated beam and $f$ is the axial force. Inserting (2) and (4) into (1), the axial and transversal equations of motion of the beam are obtained as

$$
\begin{gathered}
\frac{\partial N_{x x}}{\partial x}=I_{0} \frac{\partial^{2} u}{\partial t^{2}}-I_{1} \frac{\partial^{4} w}{\partial t^{2} \partial x^{2}}+f, \\
\frac{\partial Q_{x}}{\partial x}=I_{0} \frac{\partial^{2} w}{\partial t^{2}}+q, \\
\frac{\partial^{2} M_{x x}}{\partial x^{2}}=I_{0} \frac{\partial^{2} w}{\partial t^{2}}-I_{2} \frac{\partial^{4} w}{\partial t^{2} \partial x^{2}}+I_{1} \frac{\partial^{2} u}{\partial t^{2}}+q,
\end{gathered}
$$

where the resultant forces and moment are defined as

$$
\begin{aligned}
& N_{x x}=A_{11}\left(\frac{\partial u}{\partial x}\right)-B_{11} \frac{\partial^{2} w}{\partial x^{2}} \\
& M_{x x}=B_{11}\left(\frac{\partial u}{\partial x}\right)-D_{11} \frac{\partial^{2} w}{\partial x^{2}}
\end{aligned}
$$

and the stiffness coefficients are as

$$
\begin{gathered}
A_{i j}=\sum_{k=1}^{N_{L}} \bar{Q}_{i j}^{(k)}\left(z_{k+1}-z_{k}\right), \\
B_{i j}=\frac{1}{2} \sum_{k=1}^{N_{L}} \bar{Q}_{i j}^{(k)}\left(z_{k+1}^{2}-z_{k}^{2}\right), \\
D_{i j}=\frac{1}{3} \sum_{k=1}^{N_{L}} \bar{Q}_{i j}^{(k)}\left(z_{k+1}^{3}-z_{k}^{3}\right) .
\end{gathered}
$$

The axial and transversal loads on the beam due to moving distributed mass are as follows:

$$
\begin{aligned}
f(x, t)=- & \frac{m_{l} g}{\varepsilon}\left[H\left(x-\xi+\frac{\varepsilon}{2}\right)-H\left(x-\xi-\frac{\varepsilon}{2}\right)\right] \sin \theta, \\
q(x, t)= & \frac{-m_{l}\left(g+a_{l}\right)}{\varepsilon} \\
& \times\left[H\left(x-\xi+\frac{\varepsilon}{2}\right)-H\left(x-\xi-\frac{\varepsilon}{2}\right)\right] \cos \theta,
\end{aligned}
$$

where $a_{l}=\partial^{2} w / \partial t^{2}+2 v\left(\partial^{2} w / \partial x \partial t\right)+v^{2}\left(\partial^{2} w / \partial x^{2}\right)$ and $\xi=$ $v t+\varepsilon / 2$. In (8), $v$ is the velocity of the moving mass, $m_{l}$ is mass, $\varepsilon$ is load length, $g$ is acceleration of gravity, and $H(x)$ is Heaviside unit step function.

In a similar manner, the governing equations of the beam based on the first-order shear deformation theory can be derived in the following. For this purpose, the variation of the strain energy and the kinetic energy of the beam can be written as

$$
\begin{gathered}
\delta U=b \int_{0}^{l} \int_{-h / 2}^{h / 2}\left[\sigma_{x x}\left(\delta \varepsilon_{x x}^{(0)}+z \delta \varepsilon_{x x}^{(1)}\right)+\sigma_{x z} \delta \gamma_{x z}\right] d z d x, \\
\delta T=b \int_{0}^{l} \int_{-h / 2}^{h / 2} \rho_{0}\left[\left(\frac{\partial u}{\partial t}+z \frac{\partial \phi_{x}}{\partial t}\right)\left(\frac{\partial \delta u}{\partial t}+z \frac{\partial \delta \phi_{x}}{\partial t}\right)\right. \\
\left.+\frac{\partial w}{\partial t} \frac{\partial \delta w}{\partial t}\right] d z d x,
\end{gathered}
$$

where $\phi_{x}$ is the bending rotation of the beam cross section. The variation of strain components is as follows:

$$
\begin{gathered}
\delta \varepsilon_{x x}^{(0)}=\delta\left(\frac{\partial u}{\partial x}\right), \quad \delta \varepsilon_{x x}^{(1)}=\delta\left(\frac{\partial \phi_{x}}{\partial x}\right), \\
\delta \gamma_{x z}^{(0)}=\delta\left(\frac{\partial w}{\partial x}+\phi_{x}\right), \\
\delta \gamma_{x z}^{(1)}=0 .
\end{gathered}
$$

Inserting (9) into (1) and performing the integration by parts, one gets the following relations:

$$
\begin{gathered}
\frac{\partial N_{x x}}{\partial x}=I_{0} \frac{\partial^{2} u}{\partial t^{2}}+I_{1} \frac{\partial^{2} \phi_{x}}{\partial t^{2}}+f, \\
\frac{\partial Q_{x}}{\partial x}=I_{0} \frac{\partial^{2} w}{\partial t^{2}}+q, \\
\frac{\partial M_{x x}}{\partial x}-Q_{x}=I_{1} \frac{\partial^{2} u}{\partial t^{2}}+I_{2} \frac{\partial^{2} \phi_{x}}{\partial t^{2}},
\end{gathered}
$$

where

$$
\begin{aligned}
N_{x x} & =A_{11}\left(\frac{\partial u}{\partial x}\right)+B_{11} \frac{\partial \phi_{x}}{\partial x} \\
M_{x x} & =B_{11}\left(\frac{\partial u}{\partial x}\right)+D_{11} \frac{\partial \phi_{x}}{\partial x} \\
Q_{x} & =k A_{55}\left(\frac{\partial w}{\partial x}+\phi_{x}\right)
\end{aligned}
$$

where $Q_{x}$ transverse shear is force and $k$ is the shear correction factor. 


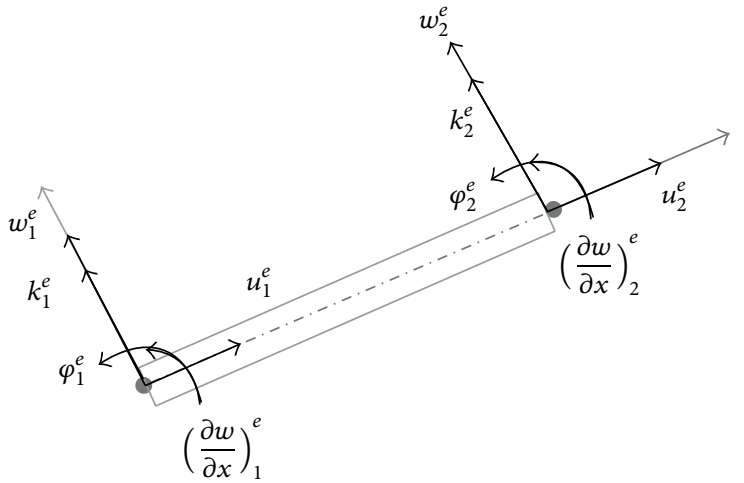

(a)

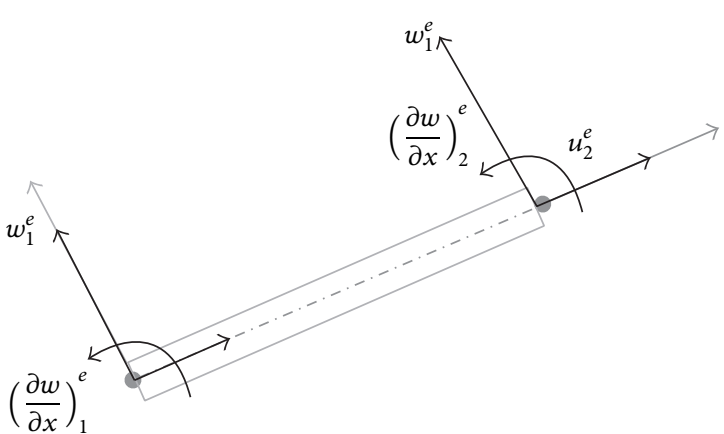

(b)

FiguRE 2: Schematics of (a) ten- and (b) six-degree-of-freedom beam elements with their primary variables $(k=\partial \varphi / \partial x$ is nodal curvature).

\section{Finite Element Formulation}

3.1. Property Matrices of Moving Distributed Mass. At any instant moving, distributed mass is on one or more beam elements depending on the beam element lengths; thus, the mass, stiffness, damping, and force matrices of moving, distributed mass are functions of time. Since moving distributed mass and beam are always in contact and moving mass slides on the beam surface, so they have the same transversal displacements while their axial displacements, are different. The governing equations of moving distributed mass in $x$ and $y$ directions are given by

$$
\begin{gathered}
F_{x}=m_{l} \ddot{u}_{x m}, \\
F_{y}=m_{l}\left(\ddot{w}+2 v \dot{w}^{\prime}+v^{2} w^{\prime \prime}\right),
\end{gathered}
$$

where $F_{x}$ is force due to friction between the moving distributed mass and inclined beam and $F_{y}$ are interaction forces between moving distributed mass and the beam. $F_{y}$ have components inertials, Coriolis, and the centrifugal forces of mass, respectively. $u_{x m}$ is axial displacement of the moving distributed mass.

3.2. Free Vibration of Symmetrically AS/3501-6 GraphiteEpoxy Laminated Beam. According to Figure 1, for an arbitrary point on the inclined beam, $u$ and $w$ displacement components in the local $x$ and $y$ directions are related to $\bar{u}$ and $\bar{w}$ in global $\bar{x}$ and $\bar{y}$ directions,

$$
\begin{aligned}
& \bar{u}=u \cos \theta-w \sin \theta, \\
& \bar{w}=u \sin \theta+w \cos \theta
\end{aligned}
$$

In the six-degree-of-freedom element (see Figure 2(b)) used in both CLT beam weak form and moving distributed mass formulations, linear Lagrange interpolation functions are used for nodal forces and displacements in $x$ direction. For its $y$ direction, cubic hermit shape functions are used as follows:

$$
\begin{gathered}
N_{1}(\zeta)=\frac{(1-\zeta)}{2}, \\
N_{2}(\zeta)=\frac{(1-\zeta)^{2}(2+\zeta)}{4}, \\
N_{3}(\zeta)=\frac{l(1-\zeta)^{2}(1+\zeta)}{8}, \\
N_{4}(\zeta)=\frac{(1+\zeta)}{2}, \\
N_{5}(\zeta)=\frac{(1+\zeta)^{2}(2-\zeta)}{4}, \\
N_{6}(\zeta)=-\frac{l(1+\zeta)^{2}(1-\zeta)}{8}, \\
\zeta=\frac{2 x}{l}-1, \quad x \in[0, l], \zeta \in[-1,1]
\end{gathered}
$$

where $N_{k}(k=1,4)$ are used for nodal force and displacements in $x$ direction. $N_{k}(k=2,3,5,6)$ are of them in $y$ direction.

On the other hand, FSDT-related ten-degree-of-freedom element (see Figure 2(a)) shape functions are the same as CLT ones. Shape functions used in FSDT rotation of cross section, $\phi_{x}$, are also cubic hermit shown in (15a).

Matrix form of equation of motion of distributed moving mass is as follows:

$$
\{F\}=[m]\{\dot{r}\}+[c]\{\dot{r}\}+[k]\{r\},
$$

where $\left\{F_{m}\right\}=\left\{\begin{array}{l}F_{x} \\ F_{y}\end{array}\right\}$ and $\{r\}=\left\{\begin{array}{c}u_{x m} \\ w\end{array}\right\}$ are the force and displacement vectors, respectively. For convenient of application of the finite element method, it is convenience to transform local 
matrices and vectors to those of global ones. Thus, according to Figure 1, the following relationships are adopted:

$$
\begin{gathered}
\{r\}=[T]\{\bar{r}\}, \\
\left\{F_{m}\right\}=[T]\left\{\bar{F}_{m}\right\},
\end{gathered}
$$

where transformation matrix of a ten-degree-of-freedom $T$ is defined as follows:

$$
T=\left[\begin{array}{cccccccccc}
\cos \theta & \sin \theta & 0 & 0 & 0 & 0 & 0 & 0 & 0 & 0 \\
-\sin \theta & \cos \theta & 0 & 0 & 0 & 0 & 0 & 0 & 0 & 0 \\
0 & 0 & 1 & 0 & 0 & 0 & 0 & 0 & 0 & 0 \\
0 & 0 & 0 & 1 & 0 & 0 & 0 & 0 & 0 & 0 \\
0 & 0 & 0 & 0 & 1 & 0 & 0 & 0 & 0 & 0 \\
0 & 0 & 0 & 0 & 0 & \cos \theta & \sin \theta & 0 & 0 & 0 \\
0 & 0 & 0 & 0 & 0 & -\sin \theta & \cos \theta & 0 & 0 & 0 \\
0 & 0 & 0 & 0 & 0 & 0 & 0 & 1 & 0 & 0 \\
0 & 0 & 0 & 0 & 0 & 0 & 0 & 0 & 1 & 0 \\
0 & 0 & 0 & 0 & 0 & 0 & 0 & 0 & 0 & 1
\end{array}\right] .
$$

Equation (16) can be rewritten in the global coordinate system as

$$
\left\{F_{m}\right\}=[\bar{m}]\{\ddot{\bar{r}}\}+[\bar{c}]\{\dot{\bar{r}}\}+[\bar{k}]\{\bar{r}\},
$$

where

$$
\begin{gathered}
{[\bar{m}]=[T]^{T}[m][T], \quad[\bar{c}]=[T]^{T}[c][T],} \\
{[\bar{k}]=[T]^{T}[k][T] .}
\end{gathered}
$$

3.3. Equation of Motion of the Entire Vibrating System. For a multiple-degree-of-freedom damped structural system, equation of motion is given by

$$
[\bar{M}(t)]\{\ddot{\bar{R}}(t)\}+[\bar{C}(t)]\{\dot{\bar{R}}(t)\}+[\bar{K}(t)]\{\bar{R}(t)\}=\{\bar{F}(t)\},
$$

where $[\bar{M}(t)],[\bar{C}(t)]$, and $[\bar{K}(t)]$ are the instantaneous overall mass, damping, and stiffness matrices, respectively, and they are time-dependent matrices. $\bar{R}(t), \dot{\bar{R}}(t)$, and $\ddot{\bar{R}}(t)$ are the displacement, velocity, and acceleration vectors, respectively, while $\bar{F}(t)$ is the instantaneous external force vector.

3.3.1. Overall Property Matrices. To take the effect of inertial and centrifugal forces of moving distributed mass into account, one must add their contribution to all property matrices of entire system. For doing this, we should add the above-mentioned moving distributed mass given by (16) and (19) to the inclined beam property matrices $\left[M_{b}\right]$ and $\left[K_{b}\right]$ that are mass and stiffness matrices, respectively. Thus, the instantaneous overall mass and stiffness matrices of the entire vibrating system are established by

$$
\begin{aligned}
{[\bar{M}(t)]_{n \times n} } & =\left[M_{b}\right]_{n \times n}+[\bar{m}]_{10 \times 10}, \\
{[\bar{K}(t)]_{n \times n} } & =\left[K_{b}\right]_{n \times n}+[\bar{k}]_{10 \times 10},
\end{aligned}
$$

where

$$
\bar{M}_{i j}=M_{b, i j}, \quad \bar{K}_{i j}=K_{b, i j} \quad(i, j=1,2, \ldots, n)
$$

except

$$
\begin{array}{r}
\bar{M}_{s_{i} s_{j}}=M_{b, s_{i} s_{j}}+\bar{m}_{i j}, \quad \bar{K}_{s_{i} s_{j}}=K_{b, s_{i} s_{j}}+\bar{k}_{i j} \\
(i, j=1,2, \ldots, 10) .
\end{array}
$$

In the last equations, $n$ represents the total degrees of freedom of the entire vibrating system and subscripts $s_{i}$ and $s_{j}(i, j=$ $1,2, \ldots, n)$ represent the numbering for the 10 degrees of freedom of the nodes of the beam elements on which the moving distributed mass applies at time $t$.

The overall damping matrix $\left[C_{b}\right]$ of the inclined beam is determined by using the theory of Rayleigh damping [17] as

$$
\left[C_{b}\right]=a[\bar{M}(t)]+b[\bar{K}(t)],
$$

where

$$
\begin{gathered}
a=\frac{2 \omega_{i} \omega_{j}\left(\xi_{i} \omega_{j}-\xi_{j} \omega_{i}\right)}{\omega_{j}^{2}-\omega_{i}^{2}}, \\
b=\frac{2\left(\xi_{j} \omega_{j}-\xi_{i} \omega_{i}\right)}{\omega_{j}^{2}-\omega_{i}^{2}} .
\end{gathered}
$$

In (25), (26a), and (26b), $[\bar{M}(t)]$ and $[\bar{K}(t)]$ are the overall mass and stiffness matrices given by $(22 a)$ and $(22 b)$, respectively, and $\xi_{i}$ and $\xi_{j}$ are damping ratios corresponding to any two natural frequencies of the structure, $\omega_{i}$ and $\omega_{j}$.

If the Coriolis force induced by the distributed moving mass is considered, one must add the contribution of the damping matrix of the moving distributed mass element, $[\bar{c}(t)]$, to the overall damping matrix of the inclined beam itself, $\left[C_{b}\right]$, to establish the instantaneous overall damping matrix, $[\bar{C}(t)]$; that is,

$$
[\bar{C}(t)]_{n \times n}=\left[C_{b}\right]_{n \times n}+[\bar{c}]_{10 \times 10},
$$

where

$$
\bar{C}_{i j}=C_{b, i j} \quad(i, j=1,2, \ldots, n)
$$

except

$$
\bar{C}_{s_{i} s_{j}}=C_{b, s_{i} s_{j}}+\bar{c}_{i j} \quad(i, j=1,2, \ldots, 10) .
$$

3.3.2. Equivalent Nodal Forces and Overall External Force Vector. The equivalent force vector induced by the moving distributed mass at any time $t$ is given by

$$
P=i P_{x}+j P_{y},
$$

where $i$ and $j$ are unit vectors in the local $x$ and $y$ directions (see Figure 3), while $P_{x}$ and $P_{y}$ are corresponding force components given by

$$
\begin{gathered}
P_{x}=-m_{l} g \sin \theta-F_{f}, \\
P_{y}=-m_{l} g \cos \theta .
\end{gathered}
$$




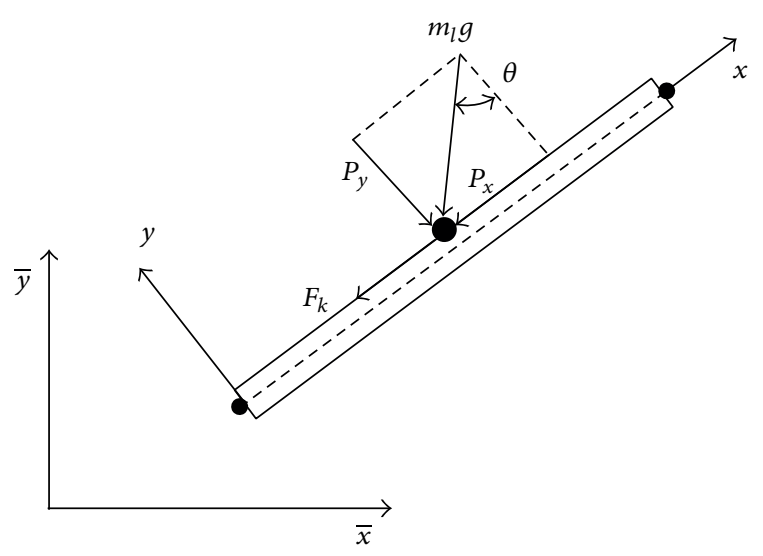

FIGURE 3: An incline beam element on which resultant moving distributed mass weight $m_{l} g$ applies.

In the last expressions, $g$ is the acceleration of gravity, $m_{l}$ is the distributed moving mass, and $\theta$ is the inclined angle of the beam. Besides, $F_{f}$ is the frictional force that is assumed to be uniformly distributed between moving distributed mass and inclined beam. The force equilibrium along the incline beam in $x$ direction requires that

$$
F_{f}=\mu m_{l} g \cos \theta=m_{l} g \sin \theta
$$

Thus,

$$
\mu=\tan \theta
$$

where $\mu$ is the friction coefficient, so (31a) should be satisfied; then, a load may move along the incline beam with a constant velocity $v$.

\section{Numerical Results and Discussion}

To obtain results given in this paper, Newark's scheme [23] has been utilized for time derivatives of (21) which are to be solved for each time interval. In this section, firstly, the convergence and accuracy of the presented formulation and the validity of obtained results are investigated. Then, the numerical results of simplified problems are presented and, whenever possible, compared to available analytical and numerical results in the literature. Finally, parametric analysis is carried out to investigate the effects of the system components properties. The shear correction factor $k$ is assumed to be equal to 5/6 in all examples.

In this section, numerical results have been presented for symmetrical four-layer AS/3501-6 clamped-clamped graphite-epoxy beams $(\theta /-\theta /-\theta / \theta)$. The results are obtained using the FSDT without Poisson effect. Table 1 shows the nondimensional fundamental frequencies $\left(\bar{\omega}=\omega L^{2} \sqrt{\rho /\left(E_{L L} h^{2}\right)}\right)$ of four-layer symmetrical angle-ply beams for clampedclamped boundary conditions. The convergence behavior

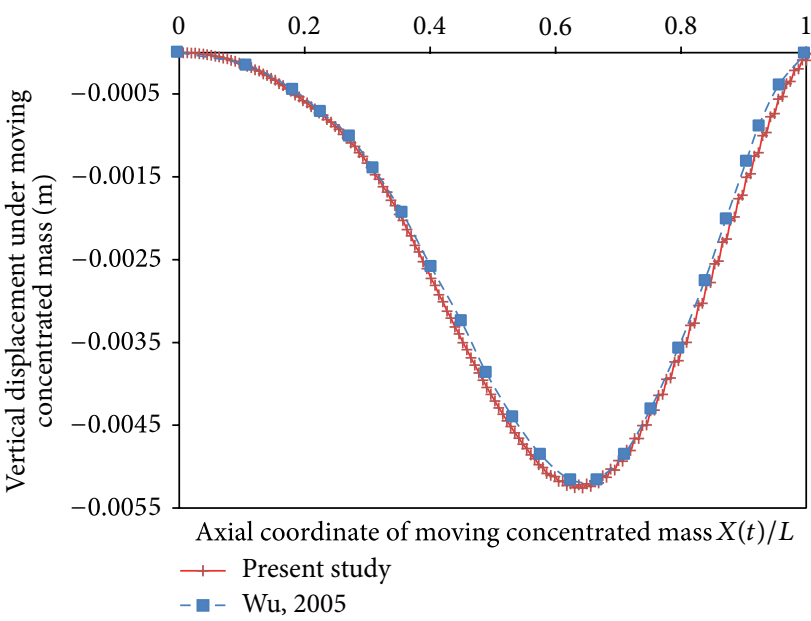

FIgURE 4: Time histories of transverse displacements of simply supported straight beam under moving concentrated mass.

and accuracy of the results for the nondimensional fundamental frequencies of different fiber orientations are presented. Physical characteristics of beam model are as follows [8]:

$$
\begin{gathered}
E_{L L}=1.448 \times 10^{11} \mathrm{pa}, \\
E_{T T}=9.653 \times 10^{9} \mathrm{pa}, \\
G_{L T}=4.137 \mathrm{pa}, \quad G_{T T}=3.448 \times 10^{9}, \\
\rho_{0}=1389.227 \mathrm{~kg} / \mathrm{m}^{3}, \quad v_{L T}=0.3 .
\end{gathered}
$$

4.1. Forced Vibration of Isotropic Straight Beam. Figure 4 shows the time histories for the transverse beam displacements of the present study and those obtained from [5]. The differences between two studies are negligible. The beam and the moving distributed mass properties are as follows [5]:

$$
\begin{gathered}
L=4.352 \mathrm{~m}, \quad b=0.018113 \mathrm{~m}, \\
h=0.072322 \mathrm{~m}, \quad \rho_{0}=15267.1756 \mathrm{~kg} / \mathrm{m}^{3}, \\
E=2020.797216 \times 10^{8} \mathrm{~N} / \mathrm{m}^{2}, \\
m_{l}=21.8 \mathrm{~kg}, \quad v=27.49 \mathrm{~m} / \mathrm{s} .
\end{gathered}
$$

4.2. Forced Vibration of Symmetrically Laminated Straight and Inclined Beam. In this section, numerical results for forced vibration of symmetrically laminated straight and inclined beam under moving distributed load are investigated. Numerical solutions performed the same beam properties as in $[8]$.

4.2.1. Straight Beam. In Figure 5, the variation of the midspan dynamic deflection of the beam divided by the maximum static deflection (DMF) is drawn versus speed ratio $\left(v / v_{\mathrm{cr}}\right)$. Comparison of DMF of this graphite-epoxy $\left(0^{\circ} / 90^{\circ} / 90^{\circ} / 0^{\circ}\right)$ composite beam under moving load is investigated using 
TABLE 1: Nondimensional fundamental frequencies $\left(\bar{\omega}=\omega L^{2} \sqrt{\rho /\left(E_{L L} h^{2}\right)}\right)$ for the AS/3501-6 graphite-epoxy of $(\theta /-\theta /-\theta / \theta)$ angle-ply clamped-clamped beams for slender ratio $L / h=15$.

\begin{tabular}{lccccccc}
\hline Laminate orientation & $0^{\circ}$ & $15^{\circ}$ & $30^{\circ}$ & $45^{\circ}$ & $60^{\circ}$ & $75^{\circ}$ & $90^{\circ}$ \\
\hline Analytical [19] & 4.8487 & 4.6635 & 4.0981 & 3.1843 & 2.1984 & 1.6815 & 1.62 \\
Kadivar and Mohebpour [7] & 4.8712 & 4.6835 & 4.1118 & 3.1908 & 2.2006 & 1.6814 & 1.6207 \\
Present study & 4.8763 & 4.6864 & 4.1050 & 3.1622 & 2.1543 & 1.664 & 1.6301 \\
\hline
\end{tabular}

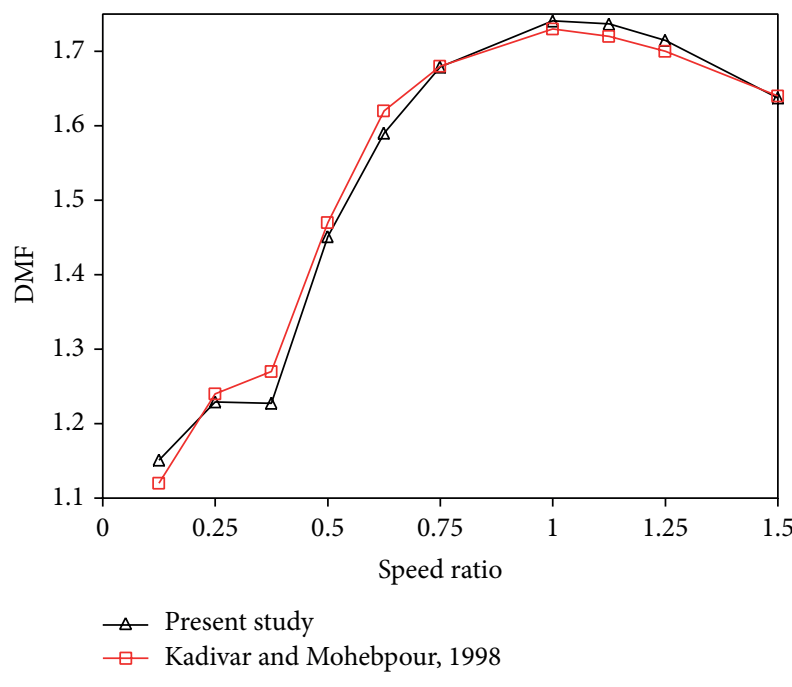

FIGURE 5: Influence of moving load velocity on dynamic magnification factor (DMF) of graphite-epoxy composite laminated beam.

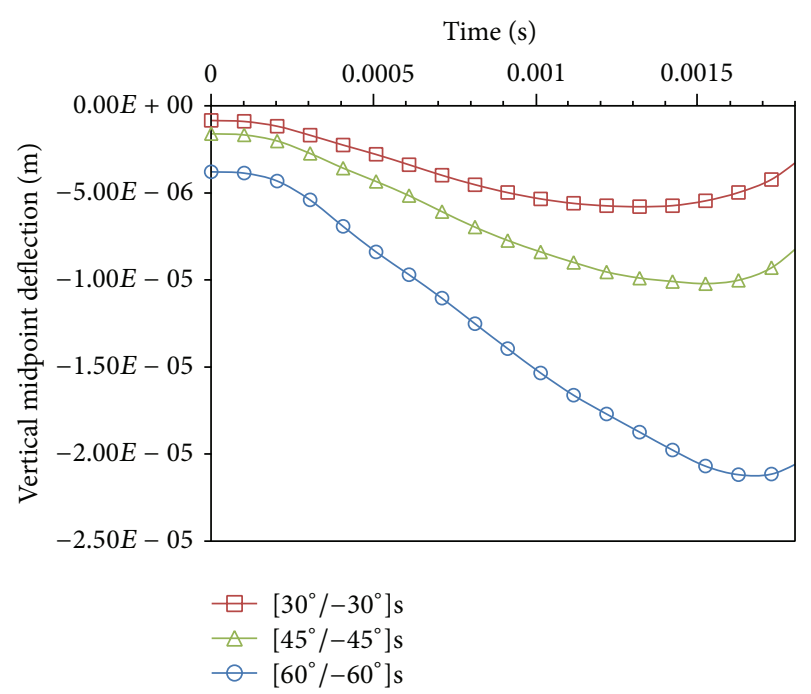

FIGURE 6: Influence of layer orientation on the transverse deflection of laminated composite beam.

CLT. As one can see, the results are in good agreement with those of [8].

(1) Influence of Layer Orientation on Transverse Deflection of Straight Beam. In Figure 6, the effect of layer orientation on the transverse deflection of straight beam based on FSDT

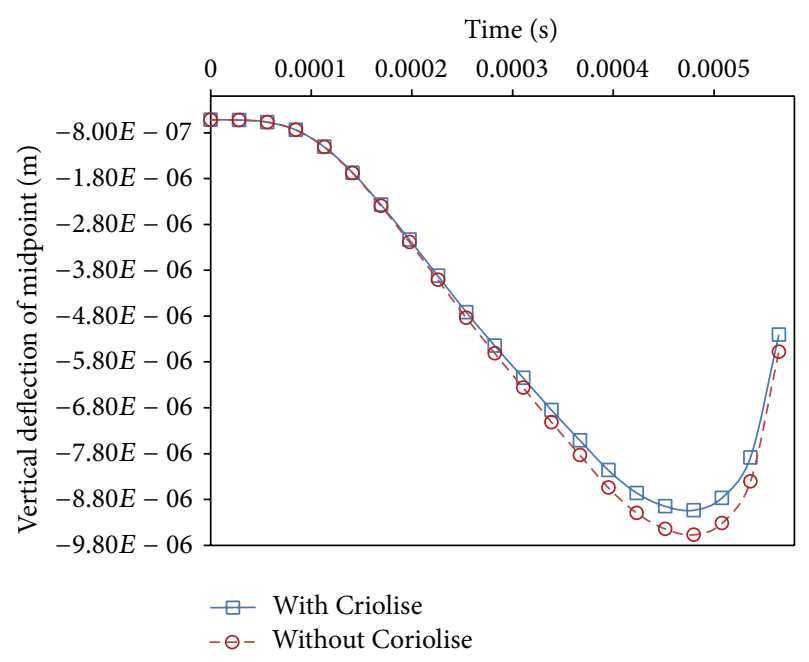

FIGURE 7: Influence of Coriolis force induced by moving distributed mass $(\varepsilon=0.01 \mathrm{~m}$ and $v=180 \mathrm{~m} / \mathrm{s})$ on transverse deflection of graphite-epoxy four-layer composite laminated beam $\left(0^{\circ} / 90^{\circ} / 90^{\circ} /\right.$ $\left.0^{\circ}\right)$.

under moving distributed mass is investigated. Load length $\varepsilon=0.01 \mathrm{~m}$, velocity $v=50 \mathrm{~m} / \mathrm{s}$, and damping ratio $\xi=$ 0.005 are assumed. As can be expected, when the fiber angle of layers changes from $60^{\circ}$ to $30^{\circ}$, the transverse deflections decrease due to increasing of the beam stiffness.

(2) Influence of the Coriolis and Centrifugal Forces of the Moving Distributed Mass on the Transverse Deflection. Figures 7 and 8 show the effect of the Coriolis $([c])$ and centrifugal $([k])$ forces induced by the moving distributed mass on the transverse deflection of beam based on the FSDT theory, respectively. It can be seen that the transverse deflections do not change considerably with considering the Coriolis and centrifugal forces. It should be noted that, with $([c] \neq 0)$ or without $([c]=0)$ the Coriolis force effect, the centrifugal force term is included in calculations and vice versa.

(3) Influence of Mass of the Moving Distributed Load on the Transverse Deflection of the Graphite-Epoxy Composite Laminated Beam. The effect of mass of moving distributed load on the transverse deflection of beam based on the FSDT is shown in Figure 9. It can be seen that the deflections considerably change with considering the mass of moving load (without its Coriolis and centrifugal effects). In addition, time when maximum deflections occurred will be delayed when the mass of load is included. 


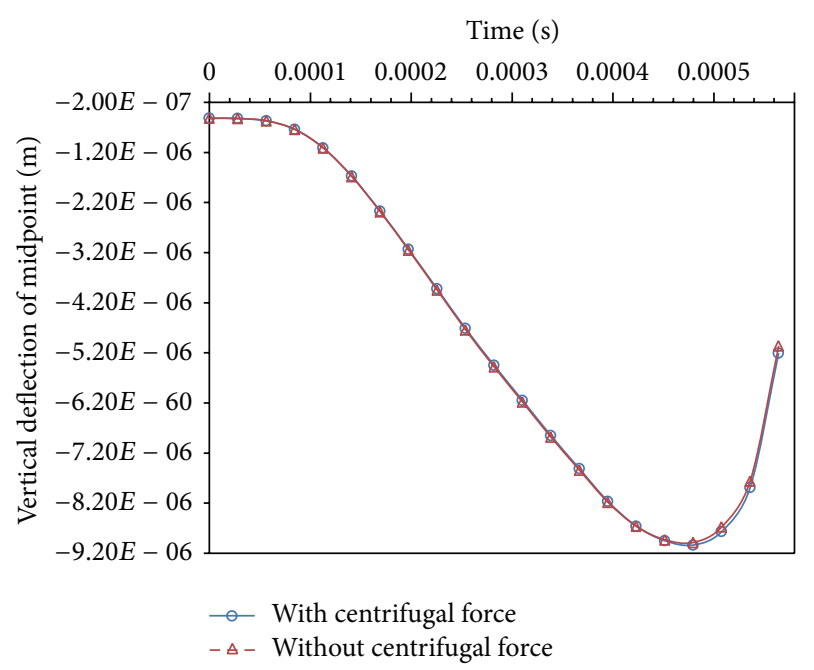

FIGURE 8: Influence of the centrifugal force induced by the moving distributed mass $(\varepsilon=0.01 \mathrm{~m}$ and $v=180 \mathrm{~m} / \mathrm{s})$ on the transverse deflection of graphite-epoxy composite laminated four-layer $\left(0^{\circ} / 90^{\circ} / 90^{\circ} / 0^{\circ}\right)$ beam.

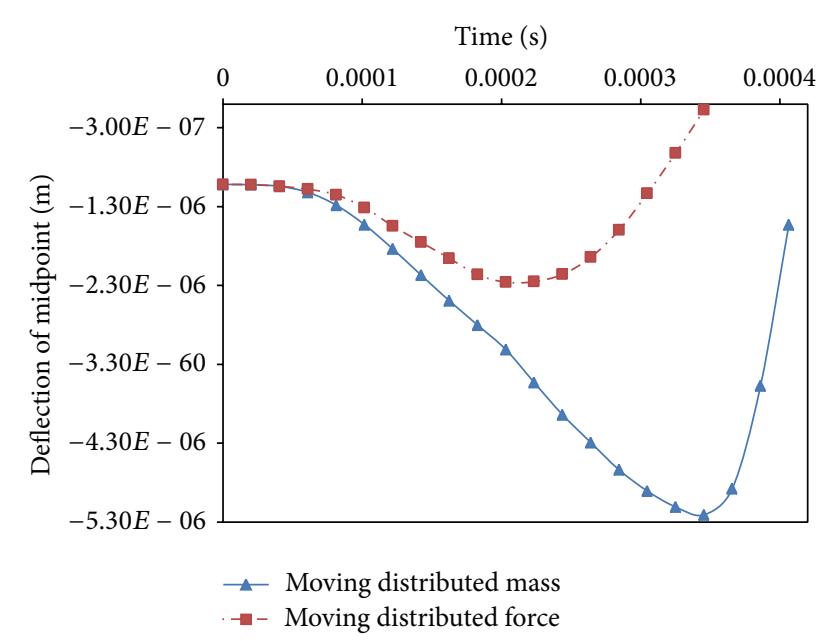

FIGURE 9: Influence of mass of the moving distributed load $(\varepsilon=$ $0.02 \mathrm{~m}$ and $v=250 \mathrm{~m} / \mathrm{s}$ ) on the transverse deflection of the graphiteepoxy four-layer composite laminated beam $\left(0^{\circ} / 90^{\circ} / 90^{\circ} / 0^{\circ}\right)$.

4.2.2. Inclined Beam. For numerical results in this section, beam properties are taken the same as [8] except the fact that the moving distributed mass is assumed to be $4.45 \mathrm{~kg}$.

(1) Influence of the Incline Angle on the Vertical and Horizontal Deflections of Graphite-Epoxy Composite Laminated Beam. Effect of the incline angle on vertical deflection of inclined beam is investigated in Figure 10. Load length $\varepsilon=0.02 \mathrm{~m}$ and velocity $v=400 \mathrm{~m} / \mathrm{s}$ are assumed. According to Figure 10, incline angle has no a significant effect on the vertical deflections.

Effect of incline angle of the beam on the horizontal deflection of incline beam is investigated in Figure 11. It can be seen that, with increasing the incline angle to $45^{\circ}$, horizontal deflection increases and maximum deflection in this angle

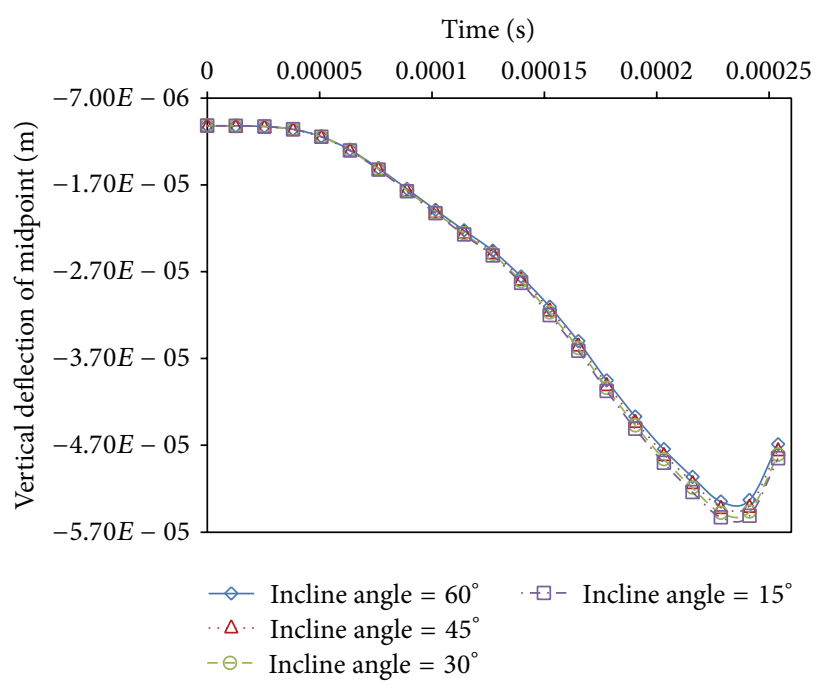

Figure 10: Influence of the incline angle on the vertical deflection of the graphite-epoxy four-layer composite laminated beam $\left(0^{\circ} / 90^{\circ} / 90^{\circ} / 0^{\circ}\right)$.

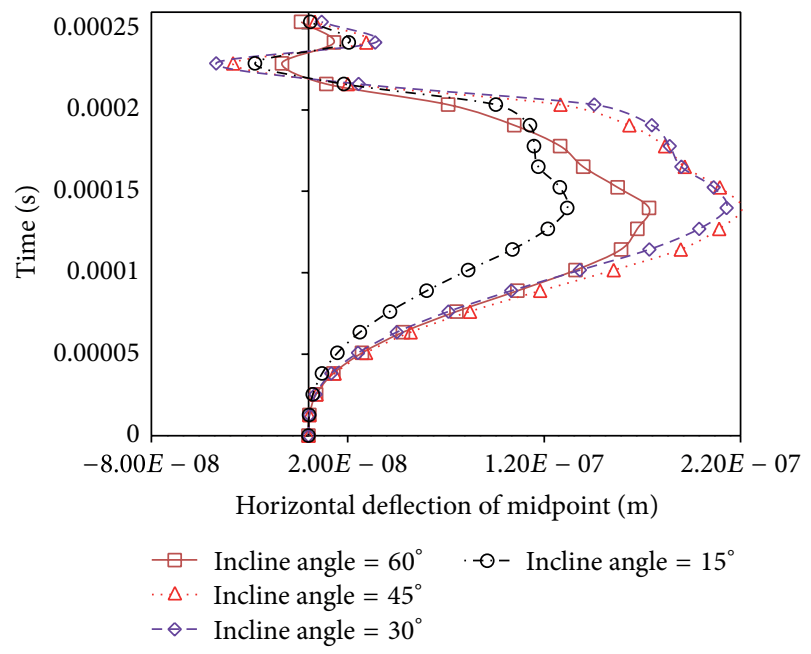

FIGURE 11: Influence of incline angle on the horizontal deflection of the graphite-epoxy four-layer composite laminated beam $\left(0^{\circ} / 90^{\circ} / 90^{\circ} / 0^{\circ}\right)$.

occurs. For angles greater than $45^{\circ}$, the horizontal deflection decreases because the frictional force decreases.

(2) Influence of Coriolis and Centrifugal Forces Induced by the Moving Distributed Mass on the Vertical and Horizontal Deflections. Figures 12 and 13 show the effect of Coriolis force of moving mass $(\varepsilon=0.02 \mathrm{~m}$ and $v=400 \mathrm{~m} / \mathrm{s})$ on vertical and horizontal deflection of inclined beam. It can be seen that Coriolis force has no considerable effect on both deflections. Centrifugal term $([k])$ is also considered in calculations.

Effect of the centrifugal force induced by the moving distributed mass on the vertical and horizontal deflections of incline beam is shown in Figures 14 and 15. It can be seen that only effect of the centrifugal force of the mass on the horizontal deflections is considerable. The Coriolis force is 


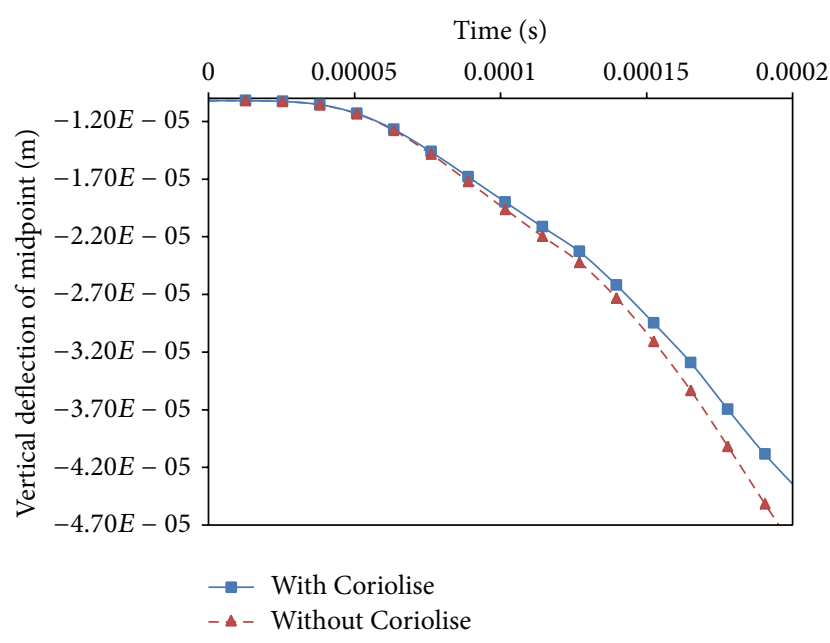

FIGURE 12: Influence of the Coriolis force on the vertical deflection of the graphite-epoxy four-layer composite laminated incline $30^{\circ}$ beam $\left(0^{\circ} / 90^{\circ} / 90^{\circ} / 0^{\circ}\right)$.

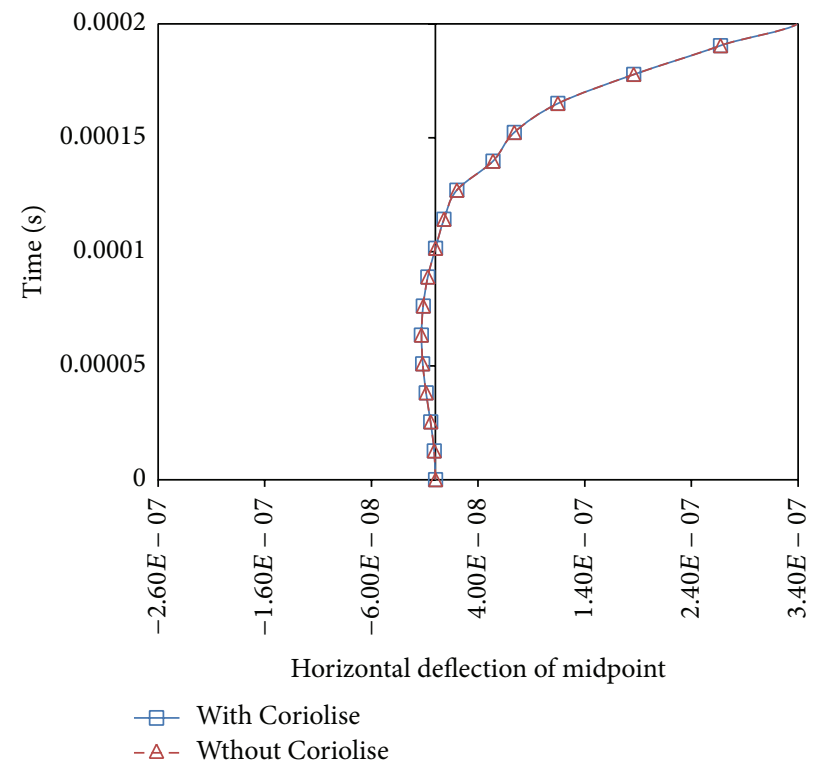

FIGURE 13: Influence of Coriolis force on horizontal deflection of graphite-epoxy four-layer composite laminated incline $30^{\circ}$ beam $\left(0^{\circ} / 90^{\circ} / 90^{\circ} / 0^{\circ}\right)$.

also considered here. Load length $\varepsilon=0.02 \mathrm{~m}$ and velocity $v=400 \mathrm{~m} / \mathrm{s}$ are assumed.

(3) Influence of the Friction Force on the Vertical and Horizontal Deflections. Figures 16 and 17 show the effect of friction force of the moving mass on the vertical and horizontal deflections of inclined beam. The friction force has considerable effect on the deflections as shown. The Coriolis and centrifugal forces are considered here. The moving mass length and velocity are $0.02 \mathrm{~m}$ and $300 \mathrm{~m} / \mathrm{s}$, respectively.

The vertical deflections of $45^{\circ}$ composite four-layers $\left(0^{\circ} / 90^{\circ} / 90^{\circ} / 0^{\circ}\right)$ beam with and without friction effect considering mass, centrifugal, and Coriolis forces are investigated

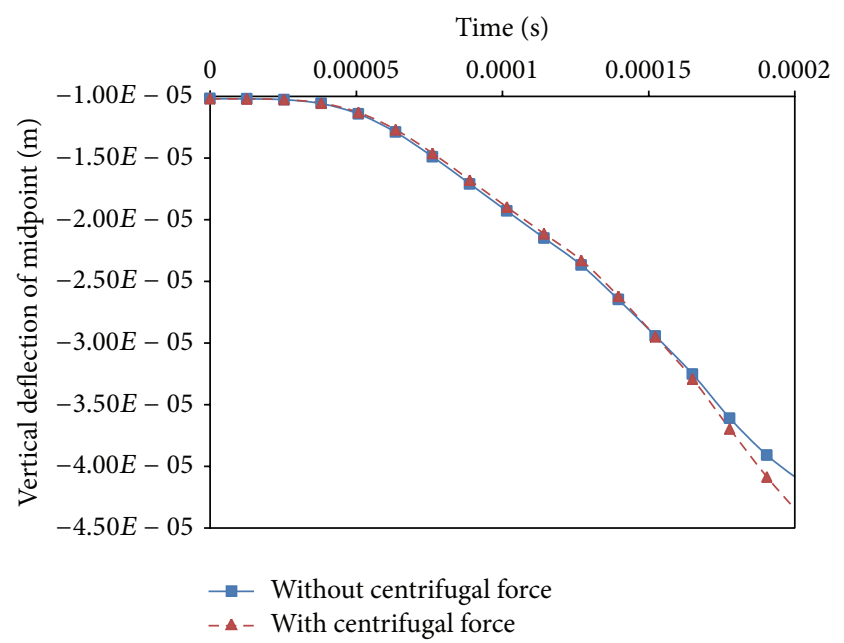

FIGURE 14: Influence of the centrifugal force on the vertical deflection of the graphite-epoxy four-layer composite laminated incline $30^{\circ}$ beam $\left(0^{\circ} / 90^{\circ} / 90^{\circ} / 0^{\circ}\right)$.

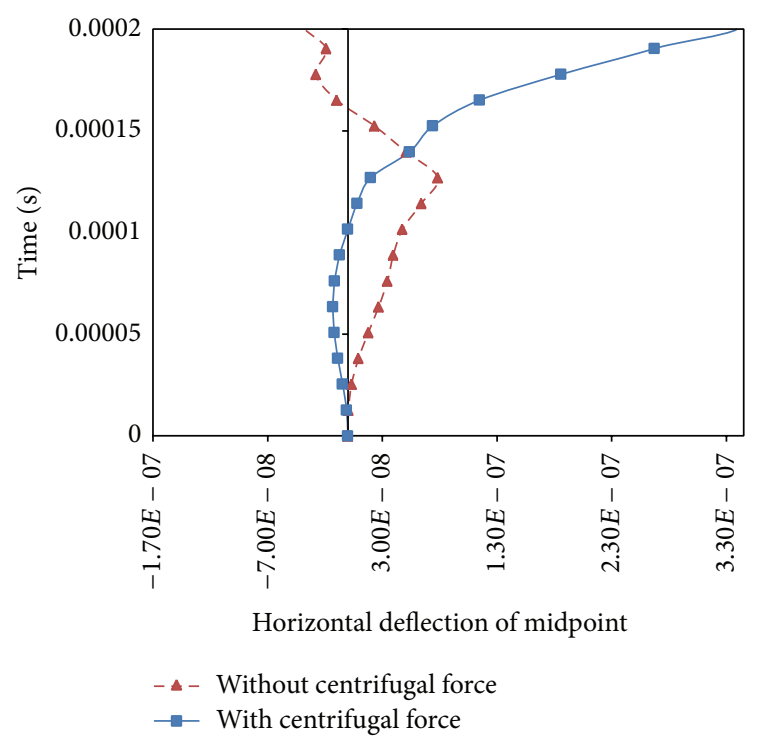

FIGURE 15: Influence of the centrifugal force on the horizontal deflection of the graphite-epoxy four-layer composite laminated incline $30^{\circ}$ beam $\left(0^{\circ} / 90^{\circ} / 90^{\circ} / 0^{\circ}\right)$.

in Figure 18. Moving mass length and velocity are $0.01 \mathrm{~m}$ and $90 \mathrm{~m} / \mathrm{s}$, respectively. As seen in the figure, the Coriolis and centrifugal forces have no effect on the vertical deflections. Effect of friction itself is not considerable but is considerable when the mass inertia is included.

(4) Layer Orientation Effect on the Deflections of the Inclined Beam. Layer orientation effect on the vertical and horizontal deflections of $45^{\circ}$ incline composite laminated beam under moving distributed mass with $\varepsilon=0.02 \mathrm{~m}, m=70.45$ and $v=250 \mathrm{~m} / \mathrm{s}$ is drawn in Figures 19 and 20, respectively. While $\left(0^{\circ} / 45^{\circ} /-45^{\circ} / 90^{\circ}\right)$ and $\left(0^{\circ} / 90^{\circ}\right)$ beams give approximately same and the smallest values for the deflections, $\left(90^{\circ} / 0^{\circ}\right)$ 


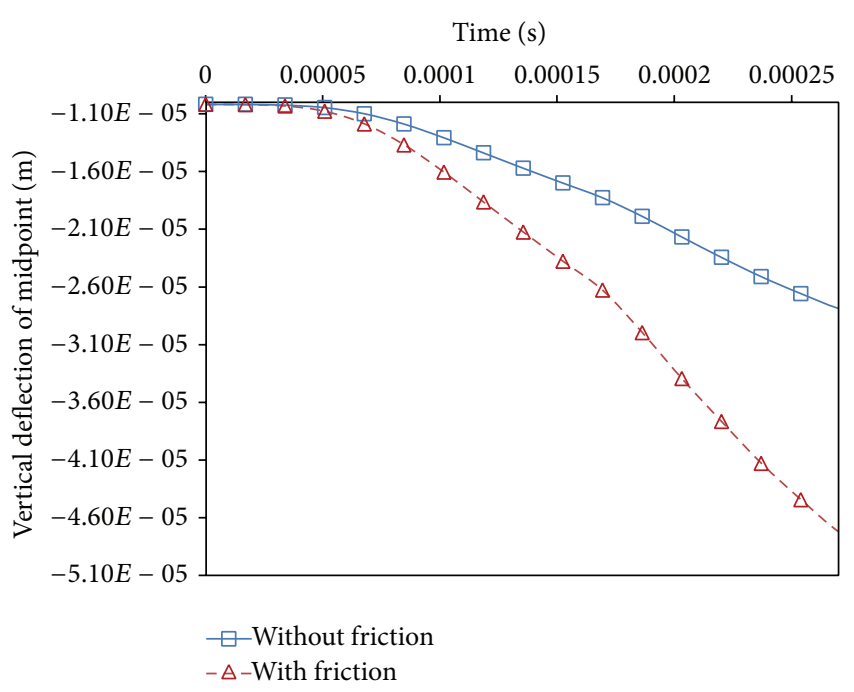

Figure 16: Influence of the friction force on the vertical deflection of the graphite-epoxy four layer composite laminated incline $45^{\circ}$ beam $\left(0^{\circ} / 60^{\circ} / 60^{\circ} / 0^{\circ}\right)$.

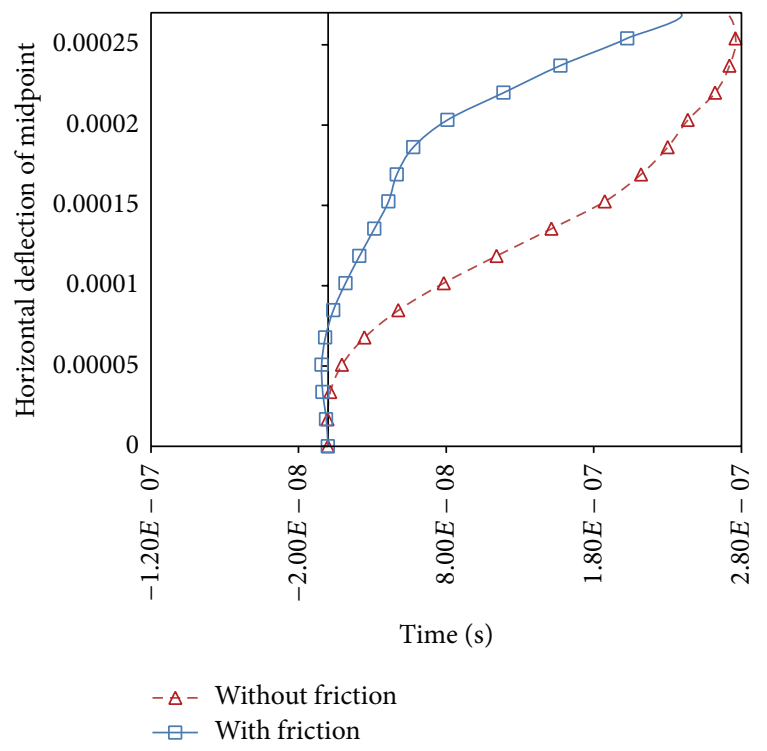

FIGURE 17: Influence of the friction force on the horizontal deflection of the graphite-epoxy four layer composite laminated incline $45^{\circ}$ beam $\left(0^{\circ} / 60^{\circ} / 60^{\circ} / 0^{\circ}\right)$.

beam the bigger values than the others due to its lower stiffness.

(5) Load Length and Mass Effects on Dynamic Magnification Factor (DMF). Effects of length and mass of moving distributed load on the DMF of $45^{\circ}$ inclined composite laminated beam are shown in Figures 21 and 22, respectively. As one can see in Figure 21, for velocities under $100 \mathrm{~m} / \mathrm{s}$ and $\varepsilon>0.01 \mathrm{~m}, \mathrm{DMF}$ is less than 1 . But, with increasing velocity to $500 \mathrm{~m} / \mathrm{s}$, DMF reaches to the maximum value (2.40). For velocities greater than $500 \mathrm{~m} / \mathrm{s}$, DMF decreases. Figure 22 shows that effect of mass only in velocities ranged

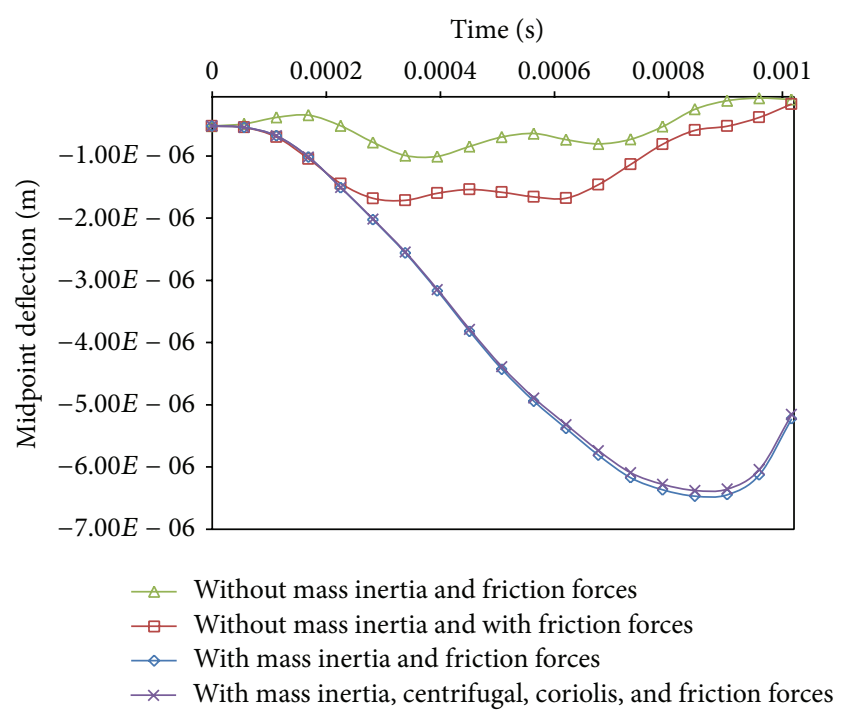

FIGURE 18: Influence of the friction force on the horizontal deflection of the graphite-epoxy four layer composite laminated incline $45^{\circ}$ beam $\left[0^{\circ} / 60^{\circ} / 60^{\circ} / 0^{\circ}\right]$.

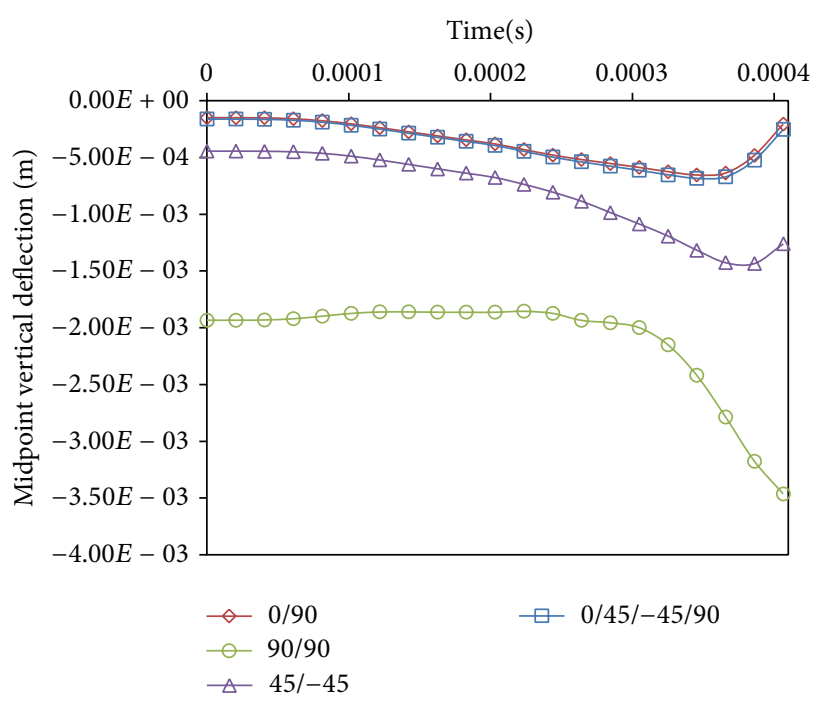

FIGURE 19: Influence of layer orientation on the vertical deflection of the incline $45^{\circ}$ composite laminated beam under moving distributed mass $(\varepsilon=0.02 \mathrm{~m}, v=250 \mathrm{~m} / \mathrm{s}$ and $m=70.45)$.

from 50 to $700 \mathrm{~m} / \mathrm{s}$ is considerable. In this velocity range, increasing velocity causes increasing in DMF and vice versa. It is important to say that the length and mass of the moving distributed load do not change the velocity that at which maximum DMF occurred; that is, $v=500 \mathrm{~m} / \mathrm{s}$.

\section{Conclusion}

The dynamic analysis of the incline laminated composite beams traversed by a moving distributed mass has been investigated. Finite element method based on the first-order shear deformation and classical theories has been used to perform the equations of motion of the beam. Comparisons 


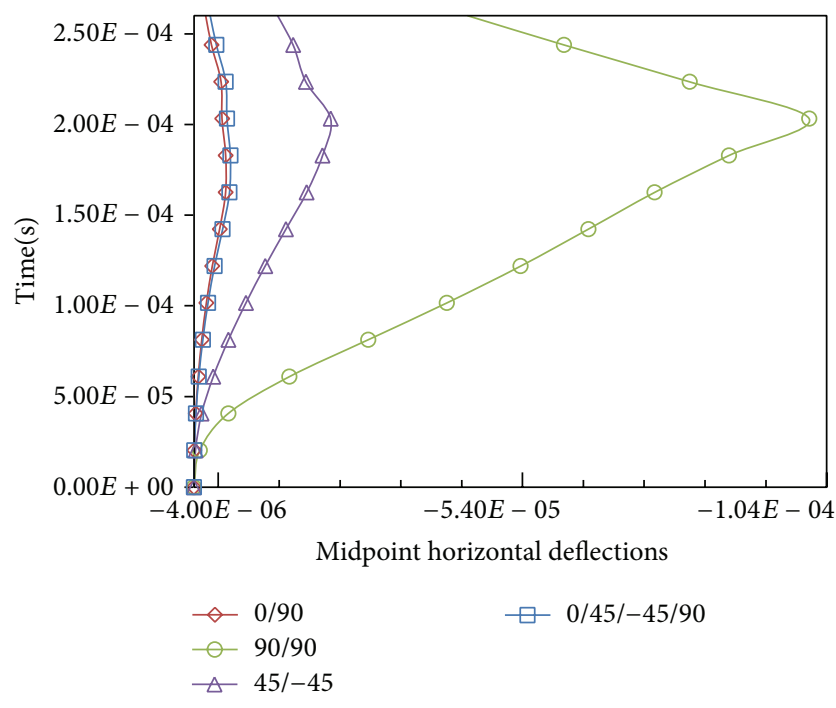

FIGURE 20: Influence of layer orientation on the horizontal deflection of the incline $45^{\circ}$ composite laminated beam under moving distributed mass $(\varepsilon=0.02 \mathrm{~m}, v=250 \mathrm{~m} / \mathrm{s}$ and $m=70.45)$.

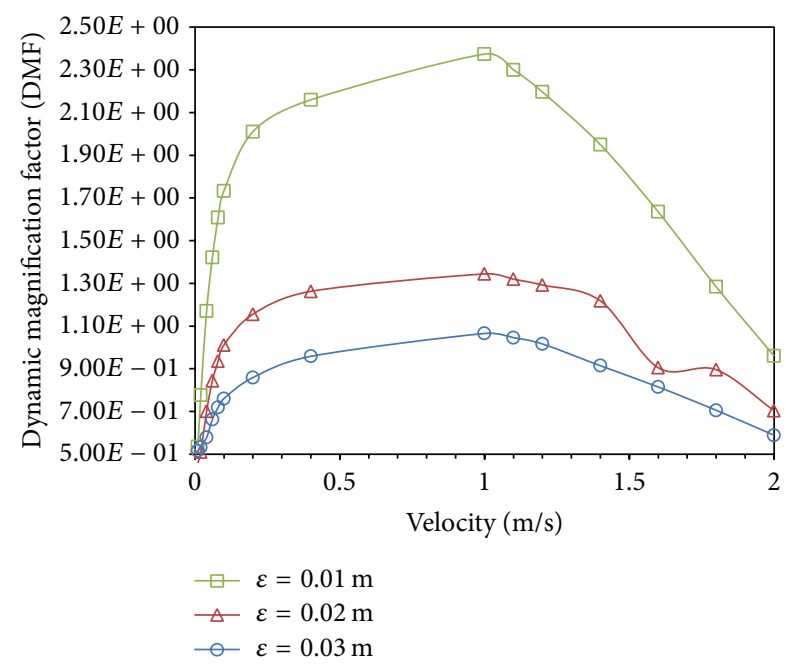

Figure 21: Load length effect on DMF of incline $45^{\circ}$ composite laminated $\left(0^{\circ} / 30^{\circ}\right)$ beam under moving distributed mass $70.45 \mathrm{~kg}$.

between the results obtained by proposed method with available results in the literature show a good agreement. It was observed that

(1) the moving mass inertial effect is considerable especially for small load lengths;

(2) moving distributed mass length and mass have no effect on critical velocity;

(3) the friction force between moving distributed mass and beam has considerable effect on deflections;

(4) maximum horizontal deflection takes place at incline angle $45^{\circ}$, where it has maximum friction coefficient;

(5) the friction effect is considerable while considering mass of moving load but the Coriolis and centrifugal

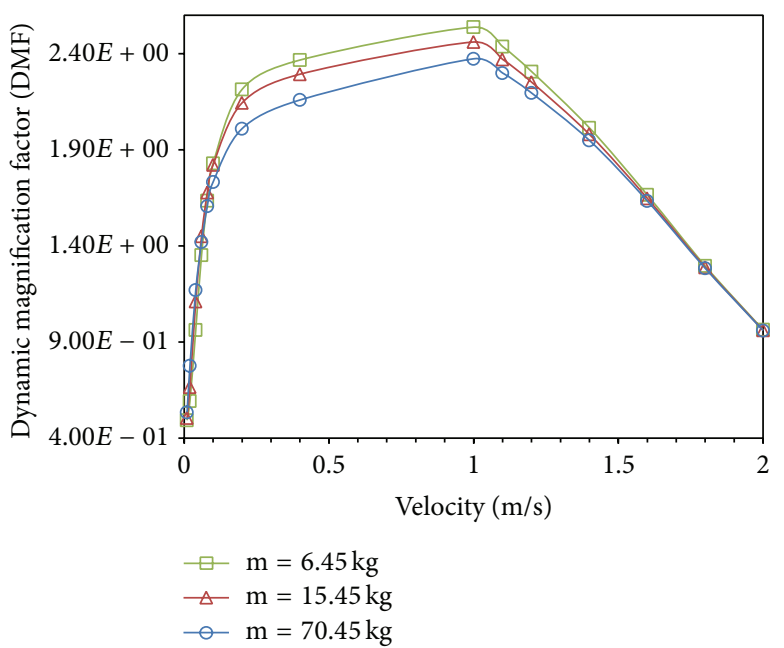

FIGURE 22: Load mass effect on DMF of incline $45^{\circ}$ composite laminated $\left(0^{\circ} / 30^{\circ}\right)$ beam under moving distributed mass $0.01 \mathrm{~m}$.

forces induced by the moving distributed mass have no effect on it;

(6) the Coriolis and centrifugal forces have no considerable effect on deflections.

\section{Conflict of Interests}

The authors declare that there is no conflict of interests regarding the publication of this paper.

\section{References}

[1] G. V. Rao, "Linear dynamics of an elastic beam under moving loads," Journal of Vibration and Acoustics, vol. 122, no. 3, pp. 281289, 2000.

[2] E. Esmailzadeh and M. Ghorashi, "Vibration analysis of a Timoshenko beam subjected to a travelling mass," Journal of Sound and Vibration, vol. 199, no. 4, pp. 615-628, 1997.

[3] R.-T. Wang, "Vibration of multi-span Timoshenko beams to a moving force," Journal of Sound and Vibration, vol. 207, no. 5, pp. 731-742, 1997.

[4] S. S. Law and X. Q. Zhu, "Study on different beam models in moving force identification," Journal of Sound and Vibration, vol. 234, no. 4, pp. 661-679, 2000.

[5] J.-J. Wu, "Dynamic analysis of an inclined beam due to moving loads," Journal of Sound and Vibration, vol. 288, no. 1-2, pp. 107131,2005

[6] K. Chandrashekhara, K. Krishnamurthy, and S. Roy, "Free vibration of composite beams including rotary inertia and shear deformation," Composite Structures, vol. 14, no. 4, pp. 269-279, 1990.

[7] M. H. Kadivar and S. R. Mohebpour, "Forced vibration of unsymmetric laminated composite beams under the action of moving loads," Composites Science and Technology, vol. 58, no. 10, pp. 1675-1684, 1998.

[8] M. H. Kadivar and S. R. Mohebpour, "Finite element dynamic analysis of unsymmetric composite laminated beams with shear effect and rotary inertia under the action of moving loads," 
Finite Elements in Analysis and Design, vol. 29, no. 3-4, pp. 259273, 1998.

[9] S. S. Law and X. Q. Zhu, "Dynamic behavior of damaged concrete bridge structures under moving vehicular loads," Engineering Structures, vol. 26, no. 9, pp. 1279-1293, 2004.

[10] S.-Y. Lee and S.-S. Yhim, "Dynamic analysis of composite plates subjected to multi-moving loads based on a third order theory," International Journal of Solids and Structures, vol. 41, no. 16-17, pp. 4457-4472, 2004.

[11] B. Kiral, Z. Kiral, and B. Baba, "Dynamic behavior of laminated composite beams subjected to a moving load," Journal of Applied Sciences, vol. 4, no. 2, pp. 271-276, 2004.

[12] J. Yang, Y. Chen, Y. Xiang, and X. L. Jia, "Free and forced vibration of cracked inhomogeneous beams under an axial force and a moving load," Journal of Sound and Vibration, vol. 312, no. 1-2, pp. 166-181, 2008.

[13] M. Şimşek and T. Kocatürk, "Free and forced vibration of a functionally graded beam subjected to a concentrated moving harmonic load," Composite Structures, vol. 90, no. 4, pp. 465473, 2009.

[14] P. Malekzadeh, M. R. G. Haghighi, and M. Gholami, "Dynamic response of thick laminated annular sector plates subjected to moving load," Composite Structures, vol. 92, no. 1, pp. 155-163, 2010.

[15] M. Şimşek, "Vibration analysis of a functionally graded beam under a moving mass by using different beam theories," Composite Structures, vol. 92, no. 4, pp. 904-917, 2010.

[16] S. M. R. Khalili, A. A. Jafari, and S. A. Eftekhari, "A mixed RitzDQ method for forced vibration of functionally graded beams carrying moving loads," Composite Structures, vol. 92, no. 10, pp. 2497-2511, 2010.

[17] S. R. Mohebpour, P. Malekzadeh, and A. A. Ahmadzadeh, "Dynamic analysis of laminated composite plates subjected to a moving oscillator by FEM," Composite Structures, vol. 93, no. 6, pp. 1574-1583, 2011.

[18] S. R. Mohebpour, A. R. Fiouz, and A. A. Ahmadzadeh, "Dynamic investigation of laminated composite beams with shear and rotary inertia effect subjected to the moving oscillators using FEM," Composite Structures, vol. 93, no. 3, pp. 1118-1126, 2011.

[19] V. Kahya, "Dynamic analysis of laminated composite beams under moving loads using finite element method," Nuclear Engineering and Design, vol. 243, pp. 41-48, 2012.

[20] M. H. Yas and M. Heshmati, "Dynamic analysis of functionally graded nanocomposite beams reinforced by randomly oriented carbon nanotube under the action of moving load," Applied Mathematical Modelling, vol. 36, no. 4, pp. 1371-1394, 2012.

[21] M. Şimşek, T. Kocatürk, and S. D. Akbaş "Dynamic behavior of an axially functionally graded beam under action of a moving harmonic load," Composite Structures, vol. 94, no. 8, pp. 23582364, 2012.

[22] A. Mamandi and M. H. Kargarnovin, "Nonlinear dynamic analysis of an inclined Timoshenko beam subjected to a moving mass/force with beam's weight included," Shock and Vibration, vol. 18, no. 6, pp. 875-891, 2011.

[23] K. J. Bathe, Finite Element Procedure in Engineering Analysis, Prentice-Hall, Englewood Cliffs, NJ, USA, 1982. 

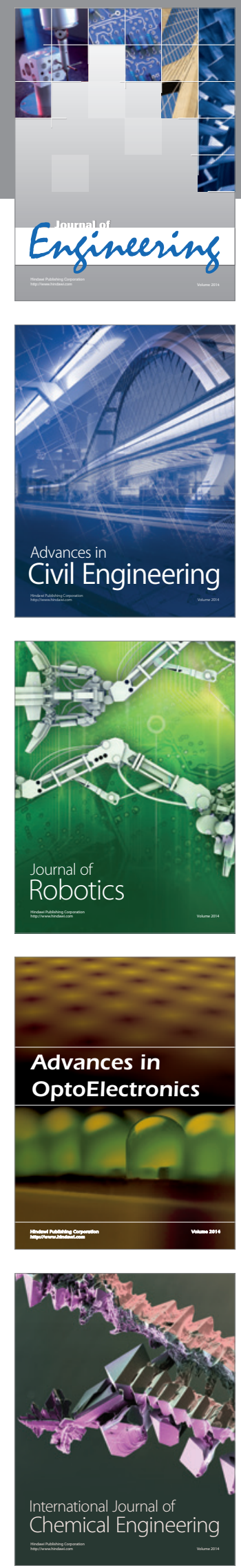

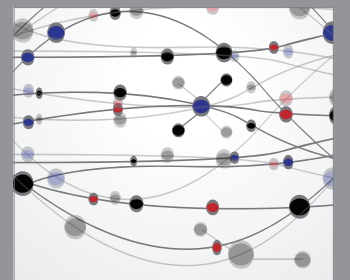

The Scientific World Journal
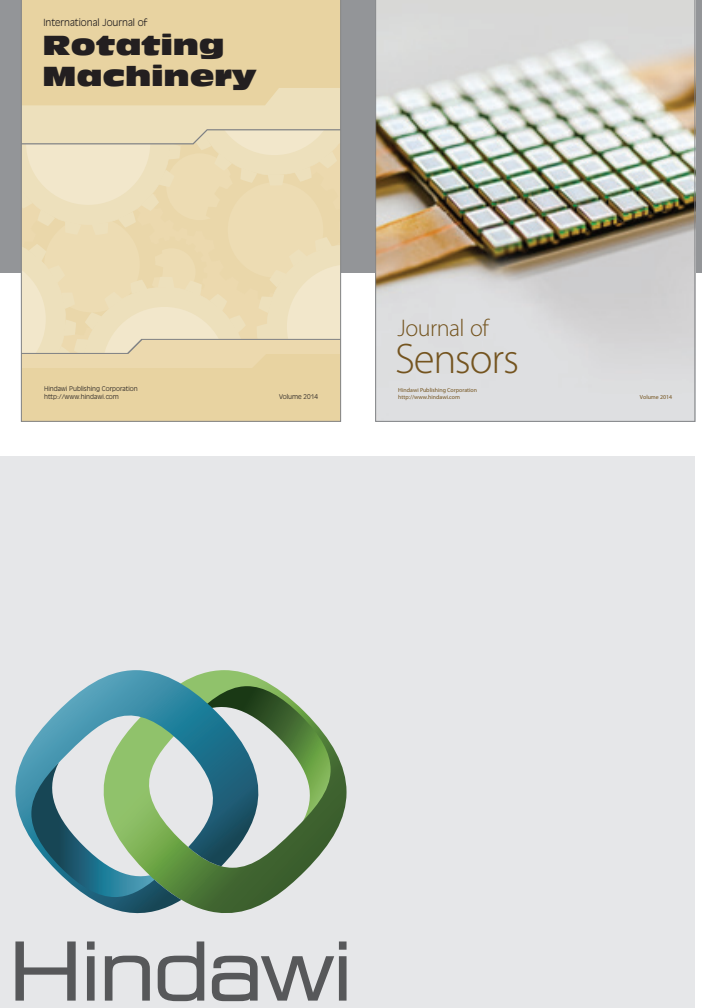

Submit your manuscripts at http://www.hindawi.com
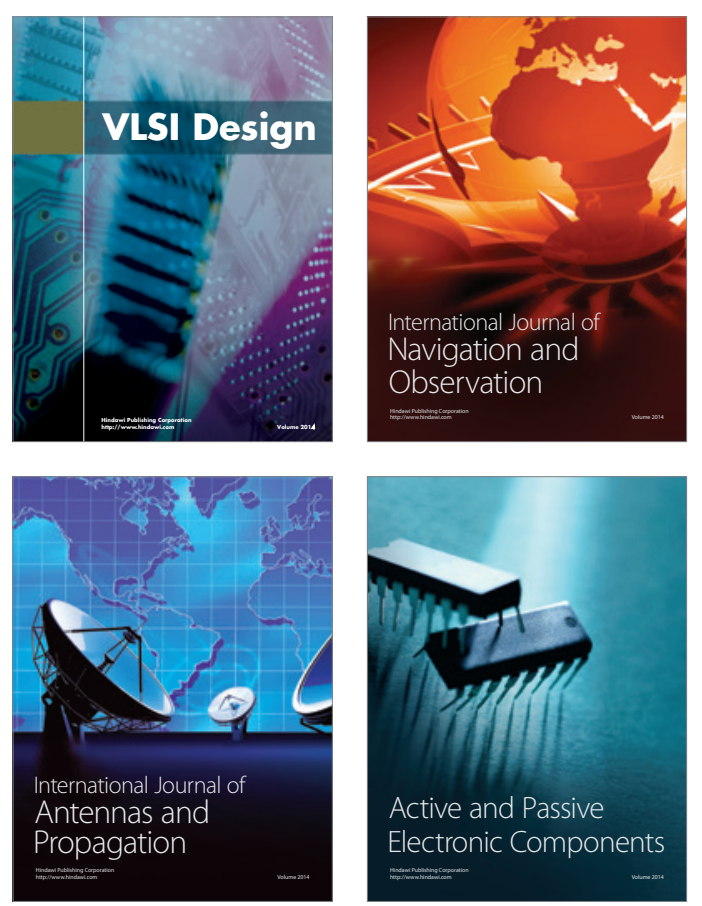
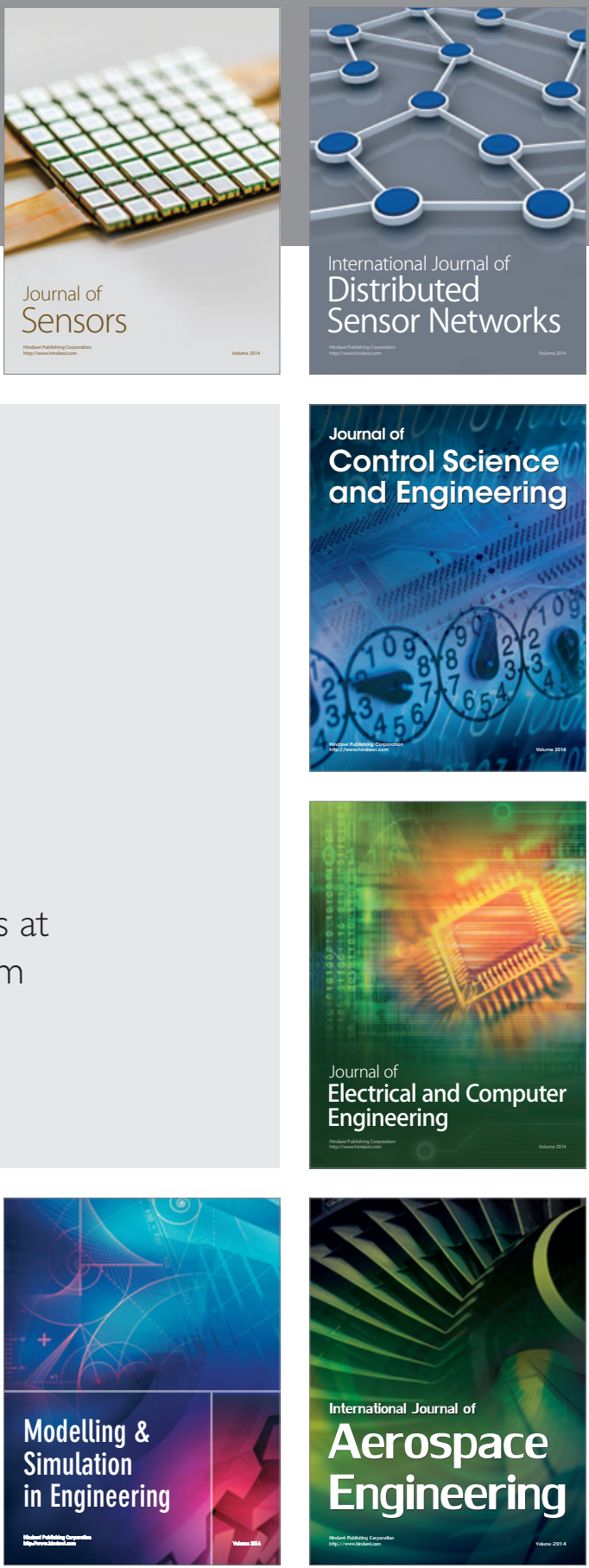

Journal of

Control Science

and Engineering
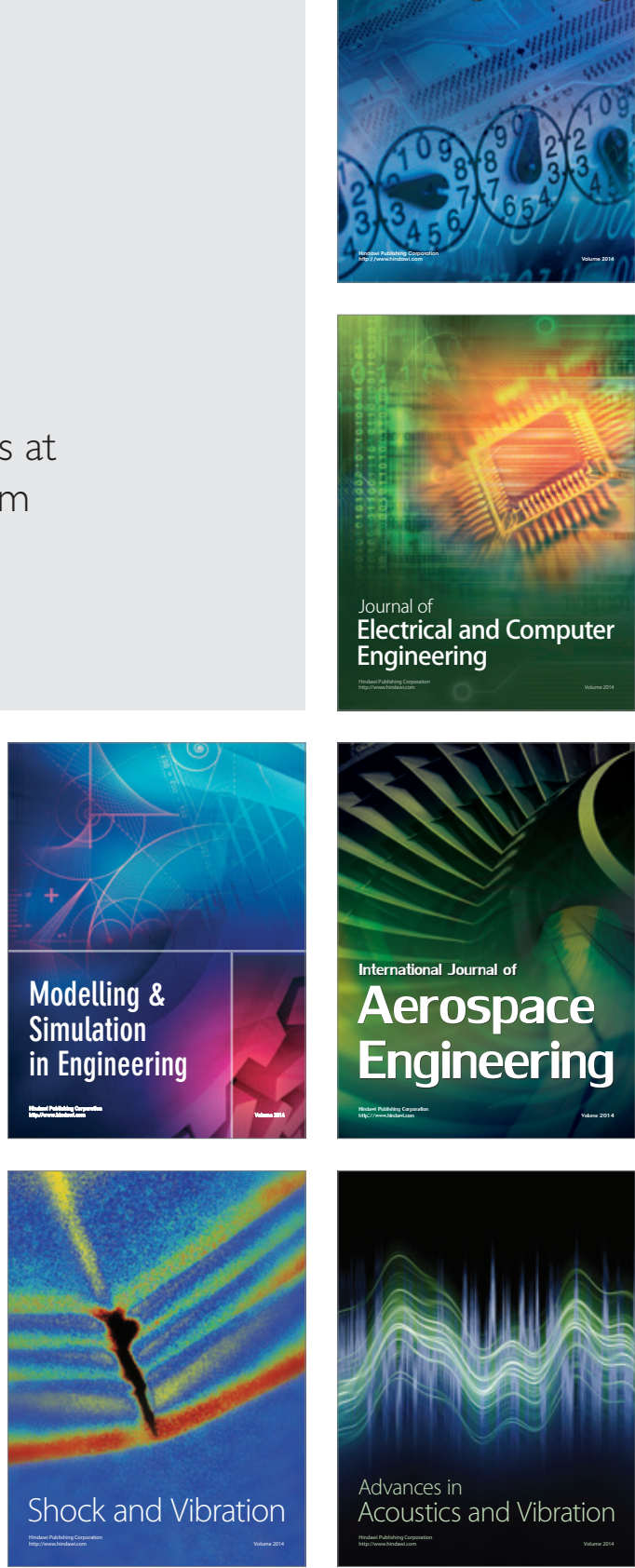